BNL-75393-2006-IR

\title{
Polyphenylenesulfied/Montomorillonite Clay Nanocomposite Coatings: Their Efficacy in Protecting Steel Against Corrosion
}

\author{
Toshifumi Sugama \\ Energy Science \& Technology Department \\ Brookhaven National Laboratory \\ Upton, NY 11973-5000 \\ Keith Gawlik \\ National Renewable Energy Laboratory \\ 1617 Cole Boulevard \\ Golden, CO 80401
}

June 2005

Notice: This manuscript has been authored by employees of Brookhaven Science Associates, LLC under Contract No. DE-AC02$98 \mathrm{CH} 10886$ with the U.S. Department of Energy. The publisher by accepting the manuscript for publication acknowledges that the United States Government retains a non-exclusive, paid-up, irrevocable, world-wide license to publish or reproduce the published form of this manuscript, or allow others to do so, for United States Government purposes. 


\section{DISCLAIMER}

This report was prepared as an account of work sponsored by an agency of the United States Government. Neither the United States Government nor any agency thereof, nor any of their employees, nor any of their contractors, subcontractors, or their employees, makes any warranty, express or implied, or assumes any legal liability or responsibility for the accuracy, completeness, or any third party's use or the results of such use of any information, apparatus, product, or process disclosed, or represents that its use would not infringe privately owned rights. Reference herein to any specific commercial product, process, or service by trade name, trademark, manufacturer, or otherwise, does not necessarily constitute or imply its endorsement, recommendation, or favoring by the United States Government or any agency thereof or its contractors or subcontractors. The views and opinions of authors expressed herein do not necessarily state or reflect those of the United States Government or any agency thereof. 


\begin{abstract}
Nanoscale montomorillonite (MMT) clay fillers became dispersed in a polyphenylenesulfied (PPS) matrix through the processes of octadecylamine (ODA) intercalation $\rightarrow$ molten PPS co-intercalation $\rightarrow$ exfoliation. Cooling this molten exfoliated material led to the formation of a PPS/MMT nanocomposite. The MMT nanofiller conferred three advanced properties on the semi-crystalline PPS: First, it raised its melting point by nearly $40^{\circ} \mathrm{C}$ to $290^{\circ} \mathrm{C}$; second, it increased its crystallization energy, implying that an excellent adherence of the nanofillers' surfaces to PPS in terms of a good interfacial bond; and, third, it abated the degree of its hydrothermal oxidation due to sulfide $\rightarrow$ sulfite linkage transformations. When this advanced PPS nanocomposite was used as a corrosion-preventing coating for carbon steel in a simulated geothermal environment at $300^{\circ} \mathrm{C}$, a coating of $\sim 150 \mu \mathrm{m}$ thickness adequately protected the steel against hot brine-caused corrosion. In contrast, an MMT-free PPS coating of similar thickness was not nearly as effective in mitigating corrosion as was the nanocompsite; in fact, the uptake of corrosive ionic electrolyte by the unmodified coating increased with an extending exposure time.
\end{abstract}

Keywords: Coatings; Corrosion and oxidation; Nanocomposites; Polymers; Geothermal 


\section{Introduction}

Polyphenylenesulfide (PPS)-based composite materials containing silicon carbide (SiC) and carbon fiber as thermal conductors exhibit a high potential as thermally conductive, anti-corrosion and-fouling liners for carbon steel heat exchanger tubes at geothermal binary-cycle power plants. In fact, our $\sim 27$ month field-validation test at the power plant operating at a brine temperature of $160^{\circ} \mathrm{C}$ gave a very promising result; the liner retained its integrity, including excellent hydrothermal stability, protection of the underlying tubes against corrosion, and minimization of silicate- and silica-related scale deposits [1-2]. This information strongly suggested that this liner would satisfactorily withstand such a harsh geothermal environment at $160^{\circ} \mathrm{C}$.

Our most recent field exposure test at the power plant was devoted to investigating the stability of the liner exposed to brine at the upgraded temperature of $200^{\circ} \mathrm{C}$. For this, we exposed PPS composite-coated carbon steel coupons to injected geothermal fluid at $200^{\circ} \mathrm{C}$ for four weeks. Post-test analyses showed that the composite coating adequately protected the carbon steel against corrosion as it did at $160^{\circ} \mathrm{C}$. More importantly; the adherence of silicate and silica scales to the coating was very weak, so that the scale layers could readily be scoured off from its surfaces using the conventional method of cleaning by hydroblasing at low hydrostatic pressure [3]. In contrast, the surfaces of the stainless steel (SS), which is commonly used for the metal components in the power plants, were vulnerable to reaction with these scales. The major factor in this undesirable happening was the formation of passive oxide layers, the corrosion resistant barrier, at the outermost surface sites of the SS. The oxide layers favorably reacted with minerals in the geothermal fluid during the exposure, so promoting the deposition of scales and the development of a strong adherence to them. Thus, despite using highpressure hydroblasting, some scales were difficult to remove compretely from the SS's surface.

Since the melting temperature of PPS is around $250^{\circ} \mathrm{C}$, it is reasonable to assume that this composite is able to withstand the brine temperatures up to $200^{\circ} \mathrm{C}$. Thus, our next biggest challenge was to apply this composite to the carbon steel plant components intended to be used at a higher brine temperatures above $250^{\circ} \mathrm{C}$. We previously studied . the hydrothermal stability of this composite coating autoclaved at $250^{\circ} \mathrm{C}$ [4]. The results 
revealed that the PPS underwent a hot brine-induced oxidation, thereby changing its molecular sulfide bridges to sulfone ones. This alteration allowed corrosive ionic species to permeate gradually through the oxidized PPS with increasing exposure time. Undoubtedly, increasing the melting temperature of PPS is an inevitable next step, if its potential application is targeted towards protecting carbon steel components at $\geq 250^{\circ} \mathrm{C}$ against corrosion and scale deposition. One specific area of application in the geothermal plants is the production wellhead, consisting of a flow line and tee, valve, and casing head, that encounter a very harsh environment with a flow velocity of $\sim 3 \mathrm{~m} / \mathrm{sec}$ of brine at $250^{\circ} \mathrm{C}$. At present, a titanium alloy-based metal is commonly used in assembling the wellhead. Hence, if this could be replaced by an inexpensive carbon steel wellhead coated with a cost-effective, anti-corrosion and -fouling PPS composite, we believe that capital expenditure would fall considerably.

In trying to enhance the melting point of PPS, our particular interest was in adapting the polymer/clay nanocomposite technology by using montomorillonite (MMT) clay as the alternative nanoscale filler. As well documented by many investigators [5-10], MMT consists of one alumina octahedral sheet sandwiched between two silica tetrahedral sheets in which the fundamental unit is a one nanometer (nm) thick, and has an approximately one hundred to several hundred nm long planar structure. Importantly, the basal space and gallery of $\sim 1.1 \mathrm{~nm}$ between the alumina and silica sheets includes various cation-exchangeable $\mathrm{Na}^{+}, \mathrm{K}^{+}, \mathrm{Li}^{+}$, or $\mathrm{Ca}^{2+}$ ions. These ions can easily exchanging with organic alkyl amine cations. Thus, the initial step of preparing a nanoscale MMT filler is the intercalation of such organic macromolecule ion exchangers containing a long alkyl chain linked to ionic amine into the gallery, thereby substantially expanding the gallery. Then, these spaces in the gallery expanded by the intercalated alkyl amine macromolecules lead to promoting the co-intercalation of polymers during curing, followed by the exfoliation of individual silicate platelets of $\sim$ one $\mathrm{nm}$ thick. Accordingly, the aspect ratio of the exfoliated MMT nanofiller in the polymer matrix is very large. As a result, such dispersion of exfoliated MMT nanofillers significantly improved some properties of the polymer including mechanical properties, thermal stability, and fire retardation [11-14]. 
Based upon this information, the emphasis of the present study was directed towards investigating the usefulness of chemically treated MMT nanofillers in enhancing the melting point of PPS. Also, it included defining the characteristics of MMT-filled PPS nanocomposite material as a corrosion-preventing coating for carbon steel in a $\mathrm{CO}_{2}$ laden brine at $300^{\circ} \mathrm{C}$.

\section{Experimental Procedures}

\section{Materials}

Montomorillonite (MMT) filler was obtained from Aldrich Chemical Inc; it included cation-exchangeable sodium, $\mathrm{Na}$. The same company also provided the octadecylamine $\left[\mathrm{CH}_{3}\left(\mathrm{CH}_{2}\right)_{17} \mathrm{NH}_{2}, \mathrm{ODA}\right]$ used as a pretreatment reagent for the MMT. The ODA was intercalated into the basal gallery of MMT in the following way. First, the two components, MMT dispersed in acidulated deionized water and the ODA dissolved in acidulated alcoholic solution, were separately prepared. For the first component, $10 \mathrm{~g}$ of MMT was added to $500 \mathrm{~g}$ acidulated deionized water containing $25 \mathrm{~g}$ of $1 \mathrm{~N} \mathrm{HCl}$, and then agitated for 10 hours at $75^{\circ} \mathrm{C}$. The resulting colloidal MMT suspension was left for 20 hours to cool to the room temperature, and then was filtered. The MMT particles retained by the filer were dried for 20 hours at $110^{\circ} \mathrm{C}$. Meanwhile, $2.5 \mathrm{~g}$ ODA was incorporated into a solution consisting of $50 \mathrm{~g}$ of isopropyl alcohol (IPA) and $31 \mathrm{~g}$ of $1 \mathrm{~N}$ $\mathrm{HCl}$, and then continuously stirred until the ODA completely dissolved in this acidulated alcoholic solution, and the solution became transparent. Afterward, $50 \mathrm{~g}$ deionized water was added to the ODA solution. Next, $6.7 \mathrm{~g}$ of dried MMT (the first component) was added to the ODA solution (the second component), and the mix was vigorously agitated. for 5 hours at room temperature to make slurry with suspended MMT particles. The suspension was filtered, and the solid particles remaining on the filtér were repeatedly washed with deionized water to eliminate any extra ODA as far as possible, without removing intercalated ones, and then dried for 24 hours at $110^{\circ} \mathrm{C}$. The dried ODA-treated MMT mass was pulverized in a vibrating sample mill for use as the filler. A thermoplastic polyphenylenesulfide (PPS) powder with a particle size of $<60 \mu \mathrm{m}$ was obtained from Ticona. A 45wt\% P.PS powder was mixed with 55wt\% isopropyl alcohol : to make a slurry coating. Then, MMT filler at $5,8,11$, and $14 \%$ by weight of the total 
amount of PPS was added to the slurry, and the mix was mechanically blended in a shear blender for 2 min to uniformly disperse the MMT particles throughout the slurry.

The carbon steel coupons were prepared in the following way. First, their surfaces were covered with a zinc phosphate ( $\mathrm{Zn} . \mathrm{Ph}$ ) primer by immersing them for $30 \mathrm{~min}$ into a phosphate solution consisting of a $5.0 \mathrm{wt} \%$ zinc orthophosphate, $10.0 \mathrm{wt} \%$ phosphoric acid, $1.0 \mathrm{wt} \%$ manganese (II) nitrate hexahydrate, and $84.0 \mathrm{wt} \%$ water at $80^{\circ} \mathrm{C}$. Then, the $\mathrm{Zn} . \mathrm{Ph}$-primed steel surfaces were rinsed with water at $25^{\circ} \mathrm{C}$, and dried in an oven at $100^{\circ} \mathrm{C}$ for $30 \mathrm{~min}$ to remove any moisture. The MMT-filled PPS coating systems were deposited on the $\mathrm{Zn}$.Ph-primed coupons as follows. The primed coupons were dipped into the slurry, and withdrawn slowly. The slurry -covered coupons were left for 20 hours at ambient temperatures to volatilize the isopropyl alcohol, and simultaneously, to promote the conversion of the slurry layer into a sintering layer. Then, the sintered layer was heated in air at $310^{\circ} \mathrm{C}$ for 3 hours to achieve melt flow, and subsequently cooled to room temperature to make a solid film. This coating process was repeated three times more to assemble coating films ranging from 120 to $150 \mu \mathrm{m}$ thick. The thickness of coating films was determined from cross-sectional examination using scanning electron microscopy (SEM).

\section{Measurements}

X-ray diffraction (XRD) was used to determine the shift in position of the basal, $d_{001}$, peaks for the "as-received" MMT and ODA-treated MMT. Differential scanning calorimetry (DSC) was employed to investigate the changes in melting and crystalline temperatures of the PPS as a function of MMT content. The structures of the untreated and treated MMT-dispersed PPS were explored using transmission electron microscopy (TEM). With Fourier-transform infrared (FT-IR), we investigated the alteration in chemical structure of the PPS containing treated and untreated MMT before and after exposure for 20 days in autoclave containing a $\mathrm{CO}_{2}$-laden brine solution $(0.5 \mathrm{wt} \%$ sodium hydrogen carbonate, $13 \mathrm{wt} \%$ sodium chloride, and $86.5 \mathrm{wt} \%$ water) at $300^{\circ} \mathrm{C}$ under a hydrothermal pressure of $8.27 \mathrm{MPa}$. The coated carbon steel panels $(62.5 \mathrm{~mm} \times 62.5$ $\mathrm{mm}$ ) were exposed for up to 20 days in an autoclave containing a $\mathrm{CO}_{2}$-laden brine solution at $300^{\circ} \mathrm{C}$. AC electrochemical impedance spectroscopy (EIS) was used to 
evaluate the ability of the exposed coating films to protect the steel from corrosion. The specimens were mounted in a holder, and then inserted into an electrochemical cell. Computer programs were prepared to calculate theoretical impedance spectra and to analyze the experimental data. Specimens with a surface area of $13 \mathrm{~cm}^{2}$ were exposed to an aerated $0.5 \mathrm{M}$ sodium chloride electrolyte at $25^{\circ} \mathrm{C}$, and single-sine technology with an input $\mathrm{AC}$ voltage of $10 \mathrm{mV}$ (rms) was employed over a frequency range of $10 \mathrm{KHz}$ to $10^{-2} \mathrm{~Hz}$. To estimate the protective performance of the coatings, the pore resistance, $\mathrm{Rp}$, $\left(\mathrm{ohm}-\mathrm{cm}^{2}\right)$ was determined from the plateau in Bode-plot scans that occurred in low frequency regions. The results from this EIS test were supported by the analysis of scanning electron microscopy (SEM) images, coupled with energy-dispersive x-ray spectrometry (EDX) of the cross-sectional area of the exposed coating layer. In addition, SEM-EDX was used to identify how well the exposed coating adheres to the $\mathrm{ZnPh}$ primer layer.

\section{Results and Discussion}

\section{ODA-treated MMT/PPS Nanocomposite}

Figure 1 depicts the XRD patterns over the diffraction range 2.21 to $0.88 \mathrm{~nm}$ for the (a) "as-received" MMT, the (b) ODA-treated MMT, and the (c) ODA-treated MMTincorporated PPS. The XRD tracing (a) of "as-received" MMT revealed a peak at 1.29 $\mathrm{nm}$, signifying that the opening between the silicate sheets in terms of basal interplanar spacing of MMT is $1.29 \mathrm{~nm}$. When the MMT was treated with the ODA, the XRD pattern (b) showed that the interplanar spacing of MMT had increased to $1.66 \mathrm{~nm}$, while the line intensity at $1.29 \mathrm{~nm}$ was strikingly attenuated. Since this increase can be accounted for by the expansion of interplanar spacing, it is reasonable to assume that the ODA, with molecular weight of 269.51, was intercalated in this spacing by ionic exchanges between the $\mathrm{Na}^{+}$in the interplanar spaces and $-\mathrm{NH}_{2}{ }^{+}$in ODA. After mixing the ODA-intercalated MMT fillers with molten PPS, we observed a considerable decay of the line intensity at $1.66 \mathrm{~nm}$ spacing (XRD tracing $\mathrm{c}$ ). This phenomenon may reflect the co-intercalation of molten PPS in the spacing, followed by the exfoliation of $\sim$ one $\mathrm{nm}$ thick silicate sheets. If this interpretation is valid, the nanoscale MMT fillers had became dispersed in the PPS matrix through the processes of ODA intercalation $\rightarrow$ 
molten PPS co-intercalation $\rightarrow$ exfoliation. Finally, cooling the material led to the formation of the PPS/MMT nanocomposite.

This information was supported by the TEM image analyses (Figure 2). Untreated and ODA-treated MMT-filled PPS solids were cut with a microtome to prepare $\sim 100 \mathrm{~nm}$ slices for TEM. Figure 2 (left) is a typical TEM image of untreated MMT-filled PPS, revealing the poor dispersion of agglomerated MMT particles in the PPS matrix. In contrast, the image of treated MMT-filled PPS sample (Figure 2, right) highlights wellseparated particles of MMT, underscoring that this sample has an exfoliated nanocomposite structure.

\section{Thermal Characterizations}

Figure 3 illustrates the cyclic DSC curve of the PPS without MMT that encompasses two endothermic- and one exothermic-transition temperatures. The former endothermic transformations included the glass-transition temperature, $T_{g}$, and the melting temperature, $T_{m}$. The latter reflected the crystallization temperature, $T_{c}$, rendering the exothermic transformation of the molten state of the polymer into a crystal state on cooling. In this curve, our attention focused on investigating the shift in the peak temperatures of $T_{m}$ and $T_{c}$ as a function of MMT content.

Figure 4 compares the $T_{m}$-related endothermic curves of $0,5,8,11$, and $14 \mathrm{wt} \%$. treated MMT-containing PPS nanocomposites. The bulk PPS noted as " $0 \%$ " had a $T_{m}$ peak of $249^{\circ} \mathrm{C}$. This single curve was converted into a doublet curve by adding $5 \mathrm{wt} \%$ MMT to the PPS; the temperatures of the first and second peaks in this doublet were $255^{\circ} \mathrm{C}$ and $268^{\circ} \mathrm{C}$, respectively. There was a further shift in both the peaks to higher temperature sites after adding $8 \mathrm{wt} \% \mathrm{MMT}$. With $11 \mathrm{wt} \% \mathrm{MMT}$, the curve's feature changed again; in particular, the second peak became the principal one, while the heat flow of first peak was strikingly attenuated. A further attenuation and growth of the first and second peaks was seen with $14 \mathrm{wt} \%$ MMT. Since the first exothermic peak is attributable to the melting point of bulk PPS, it is possible to interpret that the second peak generated at the high temperature site is associated with the melting point of PPS in the nanocomposite structure. Accordingly, the melting point of bulk PPS was 
significantly increased by adding MMT; in fact, with $14 \mathrm{wt} \% \mathrm{MMT}$, it rose by $38^{\circ} \mathrm{C}$ to $289^{\circ} \mathrm{C}$.

Figure 5 shows the changes in DSC exothermic peaks corresponding to the crystalline temperature, $T_{c}$, rendering the exothermic transformation of the molten state of PPS into its crystal state on cooling, as a function of MMT content. As seen, the $T_{c}$ tends to increase as more MMT is incorporated into the PPS. The $T_{c}$ at $175^{\circ} \mathrm{C}$ of the bulk PPS without MMT shifted to a high temperature site at $220^{\circ} \mathrm{C}$ after adding $14 \mathrm{wt} \% \mathrm{MMT}$ to the PPS. Such a shift of $T_{c}$ seemed to suggest that adding a respectable amount of MMT nanofiller promoted the extent of the melt-cooling crystallization of PPS. To support this interpretation, we determined the heat energy evolved during crystallization from the closed areas of curves with the baseline [15-16]. Figure 6 depicts the changes in crystallization energy of the PPS as a function of MMT content. As seen, the crystallization energy of the bulk PPS was conspicuously enhanced as the $11 \mathrm{wt} \% \mathrm{MMT}$ was added; beyond this content, it decreased somewhat. In exploring the interfaces between crystalable thermoplastic polymers and carbon fiber, several investigators [1719] reported that the surfaces of carbon fiber preferentially promote the nucleation of the polymer. Correspondingly, the carbon fiber-reinforced polymer had a higher nucleation density than polymer itself. They concluded that the high extent of crystallization of the polymer on the fibers' surfaces was responsible for the development of a strong interfacial bond between them. Relating our findings to these studies, the surfaces of MMT nanofillers with their large aspect ratio provided an abundance of nuclei sites for PPS crystallization. This is the major reason why the crystallization energy of PPS rose with an increasing content of MMT. Conceivably, the excellent adherence of the nanofillers' surfaces to PPS represents good interfacial bonding.

\section{Hydrothermal Oxdation}

Figure 7 shows the FT-IR spectra from the 14\% MMT-filled PPS nanocomposite film, $\sim 0.2 \mathrm{~mm}$ thick, before and after exposure for 15 days in autoclave at $300^{\circ} \mathrm{C}$. For the former, the spectrum included multiple absorption bands attributed to the two groups, phenyl rings and sulfide linkages, within the PPS, and also to another two groups in the MMT, R(Al, Mg, or Fe)-OH and silicate. The twelve bands at 3064, 3005, 1905, 1645, 
$1572,1468,1389,1179,1095,1079,1000$, and $814 \mathrm{~cm}^{-1}$ were associated with the phenyl rings, and the three bands at 743,670 , and $556 \mathrm{~cm}^{-1}$ to the sulfide linkages $[20,21]$. The remaining two bands at 3630 and $1060 \mathrm{~cm}^{-1}$ can be accounted for by the MMT-related groups, the stretching vibration of $\mathrm{O}-\mathrm{H}$ bond in the $\mathrm{R}(\mathrm{Al}, \mathrm{Mg}$, or $\mathrm{Fe})-\mathrm{OH}$ groups and the stretching modes of the Si-O bond in the silicate groups, respectively [22].

The spectrum of autoclaved film highlights the incorporation of three additional bands at 3470,1630 , and $1232 \mathrm{~cm}^{-1}$. The first two bands were due to the stretching and bending vibrations of $\mathrm{H}-\mathrm{O}-\mathrm{H}$ bond in moisture. According to the literature [23], a possible contributor to new band at $1232 \mathrm{~cm}^{-1}$ is the sulfite linkage, -O-SO-O-. Thus, an additional oxygen appears to be incorporated into the PPS film during autoclaving at $300^{\circ} \mathrm{C}$, signifying the hydrothermal oxidation of PPS.

To visualize the extent of hydrothermal oxidation of the PPS with and without MMA fillers after autoclaving for 15 days at $300^{\circ} \mathrm{C}$, the changes in the absorbance of the -O-SO-O- linkage at $1232 \mathrm{~cm}^{-1}$ and the -S- linkage at $670 \mathrm{~cm}^{-1}$ as a function of MMT content was investigated (Figure 8). The data revealed that the absorbance of the -O-SO$\mathrm{O}$ - linkage-associated band declined with an increasing content of MMT, while the $-\mathrm{S}$ linkage-related absorbance increased. With $14 \mathrm{wt} \% \mathrm{MMT}$, the absorbance of the -O-SOO- linkage was 0.017 , which was tantamount to nearly a 1.5 -fold decline compared to MMT-free PPS. This finding strongly suggested that the MMT nanofiller inhibited the oxidation of PPS brought about by the attack of $300^{\circ} \mathrm{C}$ brine.

\section{Corrosion Protection}

Our study next shifted to assessing the protection afforded by the PPS/MMT nanocomposite coatings in preventing the corrosion of carbon steel after autoclaving for up to 20 days at $300^{\circ} \mathrm{C}$. AC electrochemical impedance spectroscopy (EIS) was used to acquire this information. Figure 9 shows the overall Bode-plot curve of the bulk PPS cọating without MMT before exposure. The particular attention was paid to the impedance value in terms of the pore resistance, $R p$, which can be determined from the peak in the Bode plot occurring in a low frequency range of $10^{-1}$ to $10^{-2} \mathrm{~Hz}$. Since the $\mathrm{Rp}$ value reflects the magnitude of ionic conductivity generated by a corrosive electrolyte passing through the coating layer, a high $\mathrm{Rp}$ value means a low degree of infiltration of 
the electrolytes in the coating film, thereby resulting in a good corrosion- preventing performance of coating. Figure 10 plots the changes in Rp of the carbon steel panels (size, $60 \mathrm{~mm} \times 60 \mathrm{~mm}$ ) coated with 0, 5, 8, 11, and 14wt\% MMT-filled PPS materials against exposure times up to 20 days in a $\mathrm{CO}_{2}$-laden brine at $300^{\circ} \mathrm{C}$. Before exposure, the $\mathrm{Rp}$ value of all the coated panels ranged from a low of $5.1 \times 10^{9}$ to a high of $9.9 \times 10^{9}$ ohm- $\mathrm{cm}^{2}$. After exposing the bulk PPS coating panel noted as the " $0 \% \mathrm{MMT}$," the Rp value gradually fell, suggesting that extending the exposure time enhanced the rate of uptake of the electrolytes by the coating. A similar decline in the $\mathrm{Rp}$ value was observed from the $5 w t \%$ MMT/PPS coating. However, the rate was much lower than that of the bulk PPS coating; in fact, the Rp value of $2.6 \times 10^{9} \mathrm{ohm}-\mathrm{cm}^{2}$ for this 20-day exposed coating was nearly 2.7-fold greater than that of the bulk PPS coating at the same exposure time. In contrast, incorporating more than $8 \mathrm{wt} \%$ of MMT into the PPS impeded the permeation of electrolytes through the coating. There were no significant changes in $\mathrm{Rp}$ values for the 20-day exposed 8,11, and 14wt\% MMT-filled coatings, compared with those for these unexposed coatings, demonstrating that a proper amount of MMT nanofillers upgraded the ability of PPS coating to protect the steel against corrosion in $300^{\circ} \mathrm{C}$ brine environment. However, SEM-EDX exploration of the cross-sectional areas in the 20-day exposed $14 \mathrm{wt} \% \mathrm{MMT}$ coating revealed that some brine had permeated its superficial layer (Figure 11). As is evident from EDX spectrum, a layer extending from the outermost surface to a depth of $\sim 25 \mu \mathrm{m}$, denoted as area " $\mathrm{A}$ ", included the brinerelated $\mathrm{Na}$ element; the other elements, $\mathrm{S}, \mathrm{Si}, \mathrm{Al}, \mathrm{O}$, and $\mathrm{C}$, are derived directly from the PPS and MMT. No Na was detected at the location marked as area "B", that is $\sim 50 \mu \mathrm{m}$ below the surface. With this limited information, there is no clear whether the extent of infiltration of the brine increases with extended exposure times.

One important issue governing the maximum effectiveness of coating in preventing the corrosion of steel is its good adherence to the primer. Poor adherence often causes the generation of blisters where the brine permeates through the coating layers. The ideal mode of adherence is that bond failure at interfaces occurs in either the coating or primer layers. To obtain this information, the 20-day exposed coating layer was removed physically from the $\mathrm{Zn}$.Ph-primed steel surfaces, and then the interfacial site of coating was explored by SEM-EDX to identify the locus of the bond failure at the 
interfaces between them (Figure 12). The SEM image revealed that the crystalline $\mathrm{Zn}$. $\mathrm{Ph}$ primer layer adhered well to the coating, clearly verifying that interfacial bond failure took place in the $\mathrm{Zn}$. Ph primer layer. This cohesive failure mode can be taken as evidence that the coating was tightly linked to the primer $_{j}$ and also, that the strength of interfacial bond was much greater than that of the primer itself.

\section{Conclusion}

The cation- exchange reaction between octadècylamine (ODA) and montomorillonite (MMT) clay led to the intercalation of ODA into the $\sim 1.1 \mathrm{~nm}$ gallery within the MMT structure. Further, this intercalation played an important role in promoting the exfoliation of individual nanoscale silicate plates formed by the cointercalation of molten polyphenylensulfide (PPS), thereby generating a nanoscale MMTfilled PPS nanocomposite. Exfoliation not only uniformly dispersed the MMT nanofillers in the PPS matrix, but also conferred three advanced properties on semi-crystalline PPS polymer; 1) an increase in its melting temperature by nearly $40^{\circ}$ to $\sim 290^{\circ} \mathrm{C}, 2$ ) enhanced crystallization energy, reflecting the development of a good interfacial bond between nanofiller and PPS, and, 3) abatement of its hydrothermal oxidation attributed to the transformation of sulfide $\rightarrow$ sulfite linkage. When this advanced PPS/MMT nanocomposite was used as the high temperature corrosion-preventing coating of zinc phosphate ( $\mathrm{Zn} . \mathrm{Ph}$ )-primed carbon steel, a coating of $~ 150 \mu \mathrm{m}$ thickness adequately mitigated corrosion of steel during an exposure for 20 days in a $300^{\circ} \mathrm{C}$ brine environment. In fact, there were no significant changes in the coating's pore resistance, $\mathrm{Rp}$, that represents the extent of the uptake of corrosive ionic species. In contrast, the Rp value of the coating without MMT declined with increasing exposure time, meaning that

prolonging the exposure time enhanced the extent of infiltration of corrosive ionic species through the coating layer. Also, the adherence of the exposed nanocomposite coating to the $\mathrm{Zn} . \mathrm{Ph}$ primer was outstanding, as verified by the fact that the bond failure at interfaces between the coating and primer took place in the primer layer.

\section{References:}

[1] T. Sugama, D. Elling, K. Gawlik, J. Mater. Sci. 37 (2002) 4871. 
[2] K. Gawlik, T. Sugama, Geothermal Resources Council Transactions, 24 (2003) 450.

[3] T. Sugama, K. Gawlik, Geothermal Resources Council Transactions, 28 (2004) 365.

[4] T. Sugama, K. Gawlik, Polym. Polym. Compos. 11 (2003) 161.

[5] Y. Komori, K. Kuroda, in: T.J. Pinnavaia and G.W. Beall (Eds.), Polymer-Clay Nanocomposites, John Wiley \& Sons, Ltd., New York, 2000, pp. 3-18.

[6] E. Ruiz-Hitzky, P. Aranda, in: T.J. Pinnavaia"and G.W. Beall (Eds.), PolymerClay Nanocomposites, John Wiley \& Sons, Ltd., New York, 2000, pp. 18-46.

[7] C. Chen, D. Curliss, Polym. Materi. Sci. \& Eng. 88 (2002) 88.

[8] A. Goldstein, M. Beer, J. Euro. Ceram. Soc. 24 (2004) 3187.

[9] J.Y. Lee, H.K. Lee, Mater. Chem. Phys. 85 (2004) 410.

[10] M. Pospisil, A. Kalendova, P. Capkova, J. Simonik, M. Valaskova, Colloid Interface Sci. 277 (2004) 154.

[11] A.B. Morgan, J.W. Gilman, R.H. Harris, C.L. Jackson, C.A. Wilkie, J. Zhu, Polym. Materi. Sci. \& Eng. 83 (2000) 53.

[12] A. Tidjani, C.A. Wilkie, Polym. Degrad. Stab., 74 (2001) 33.

[13] B. Jurkowski, Y.A. Olkhov, Thermochimica Acta, 414 (2004) 243.

[14] C. Lam, H. Cheung, K. Lau, L. Zhou, M. Ho, D. Hui, Composites: Part B, 36 (2005) 263.

[15] C.S. Ray, W. Huang, D.E. Day, J. Am. Ceram. Soc. 74 (1991) 60.

[16] K.F. Kelton, J. Am. Ceram. Soc. 75 (1992) 2449.

[17] A.J. Waddon, M.J. Hill, A. Keller, D.J. Blundell, J. Mater. Sci. 22 (1987) 1773.

[18] X.J. Qian, S.E. Rickert, J.B. Lando, J. Mater. Res. 4 (1989) 1005.

[19] T.Q. Li, M.Q. Zhang, K. Zhang, H.M. Zeng, Polymer, 41 (2000) 161.

[20] R.W. Lenz, C.E. Handlovits, J. Polym. Sci. 53 (1960) 167.

[2.1] A.B. Port, R.H. Still, J. Appl. Polym. Sci. 24 (1979) 11.45.

[22] V.C. Farmer, J.D. Russell, Spectrochimica Acta, 20 (1964) 1149.

[23] L.J. Bellamy, The infrared spectra of complex molecules, Chapman and Hall, London, 1975, pp. 349-410. 


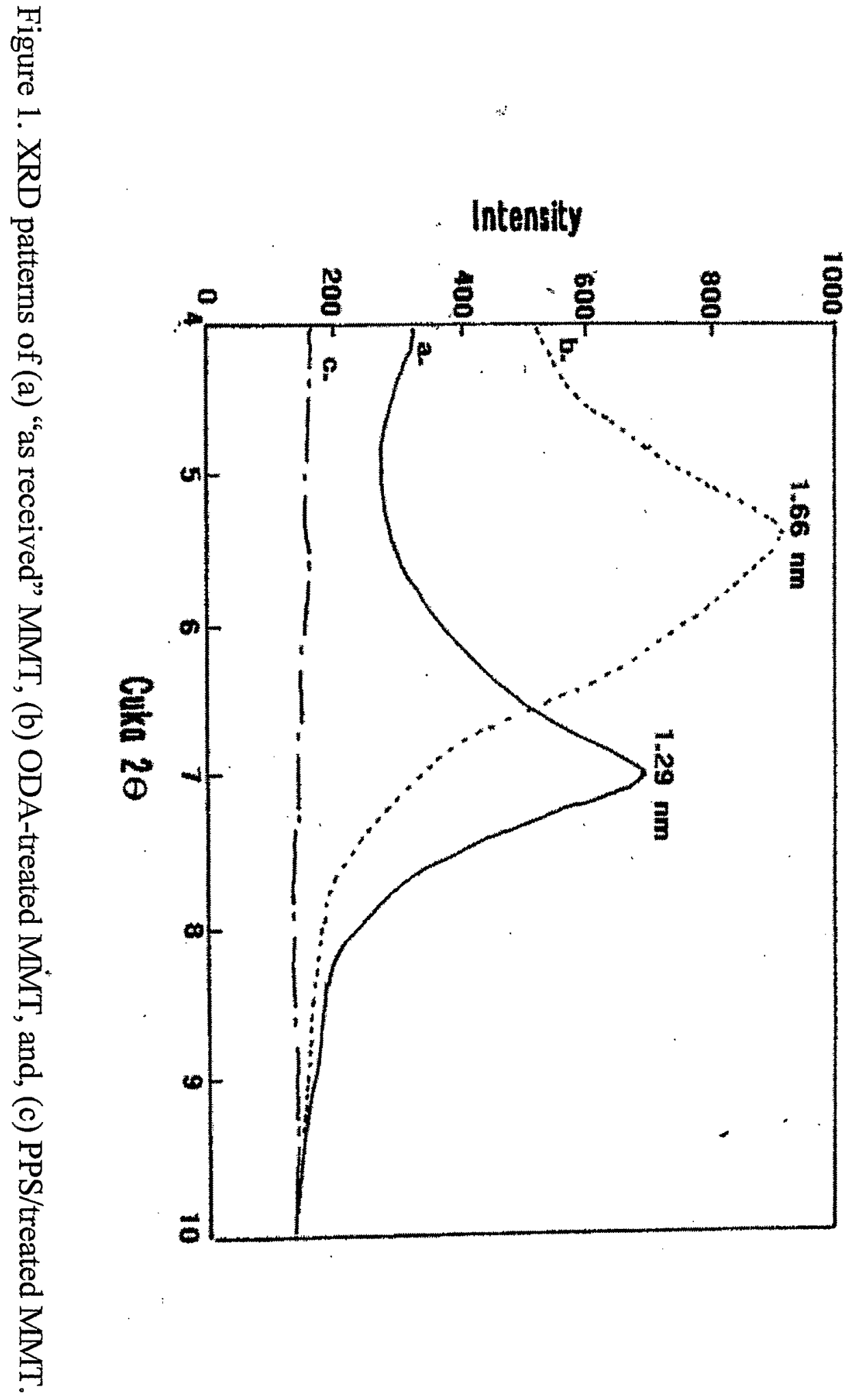




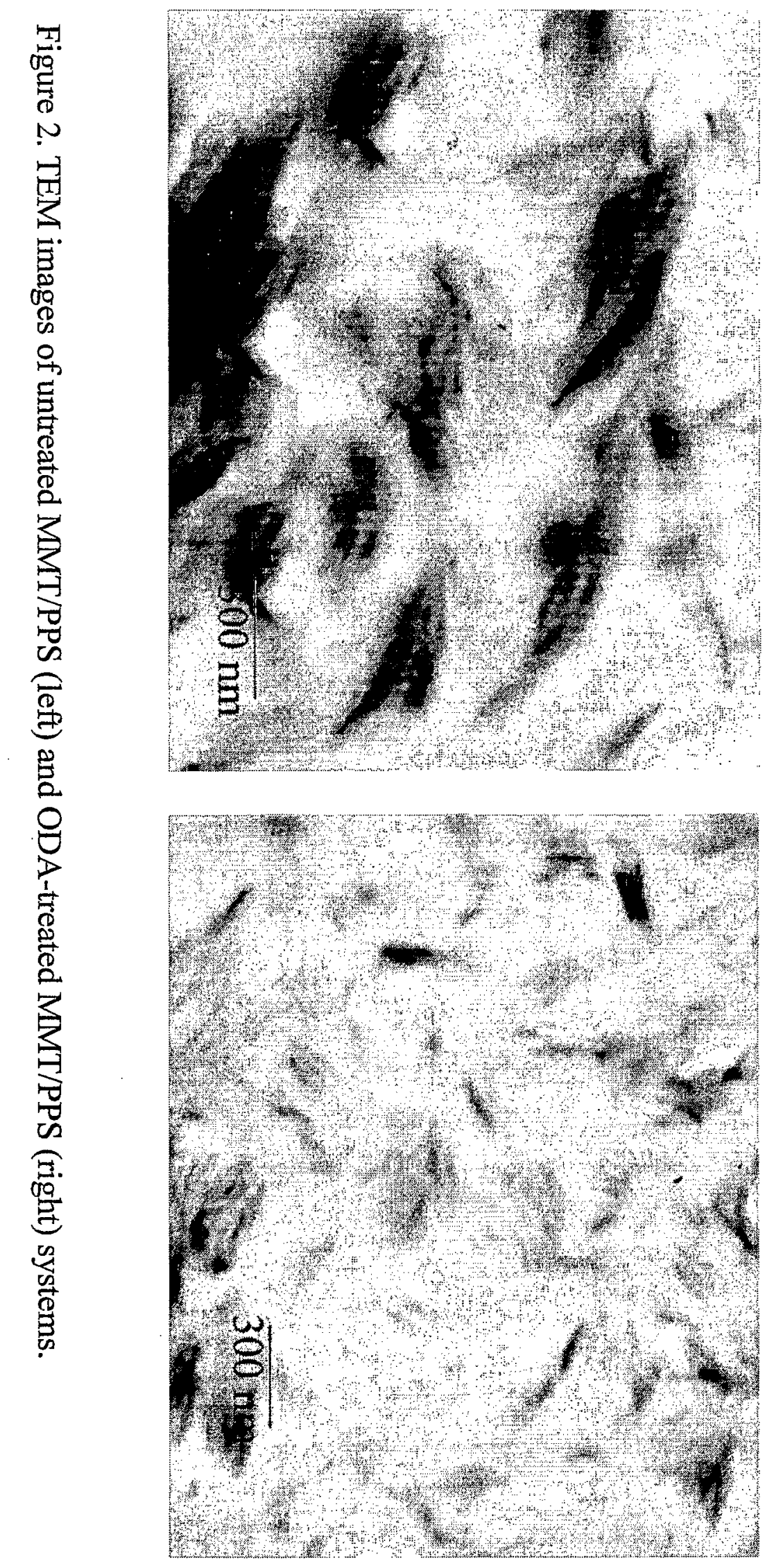




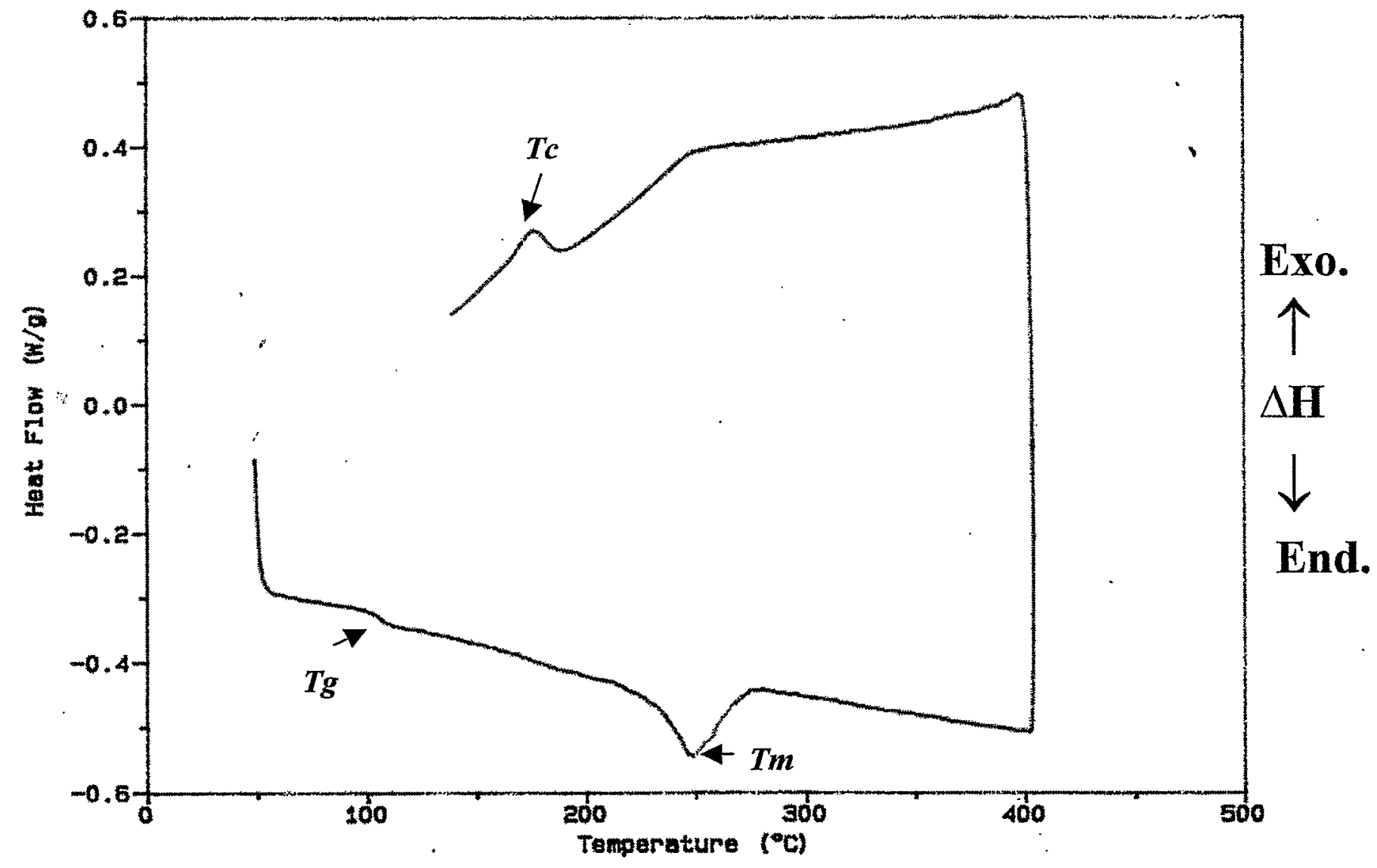

Figure 3. Typical cyclic DSC curve encompassing the glass transition temperature, $\mathrm{Tg}$, endothermic melting point, $T m$, and exothermic crystallization temperature for semi-crystalline PPS. 


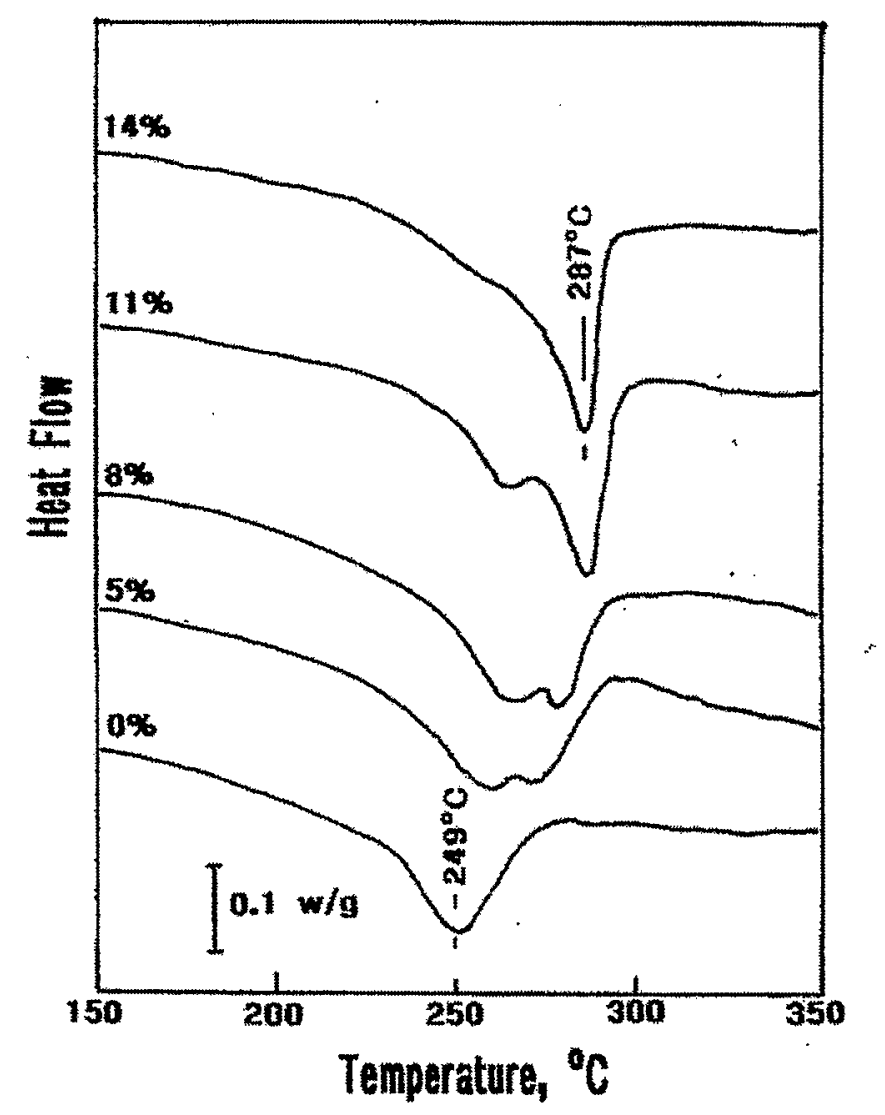

Figure 4. Shift in the melting temperature of PPS as a function of the content of treated MMT. 


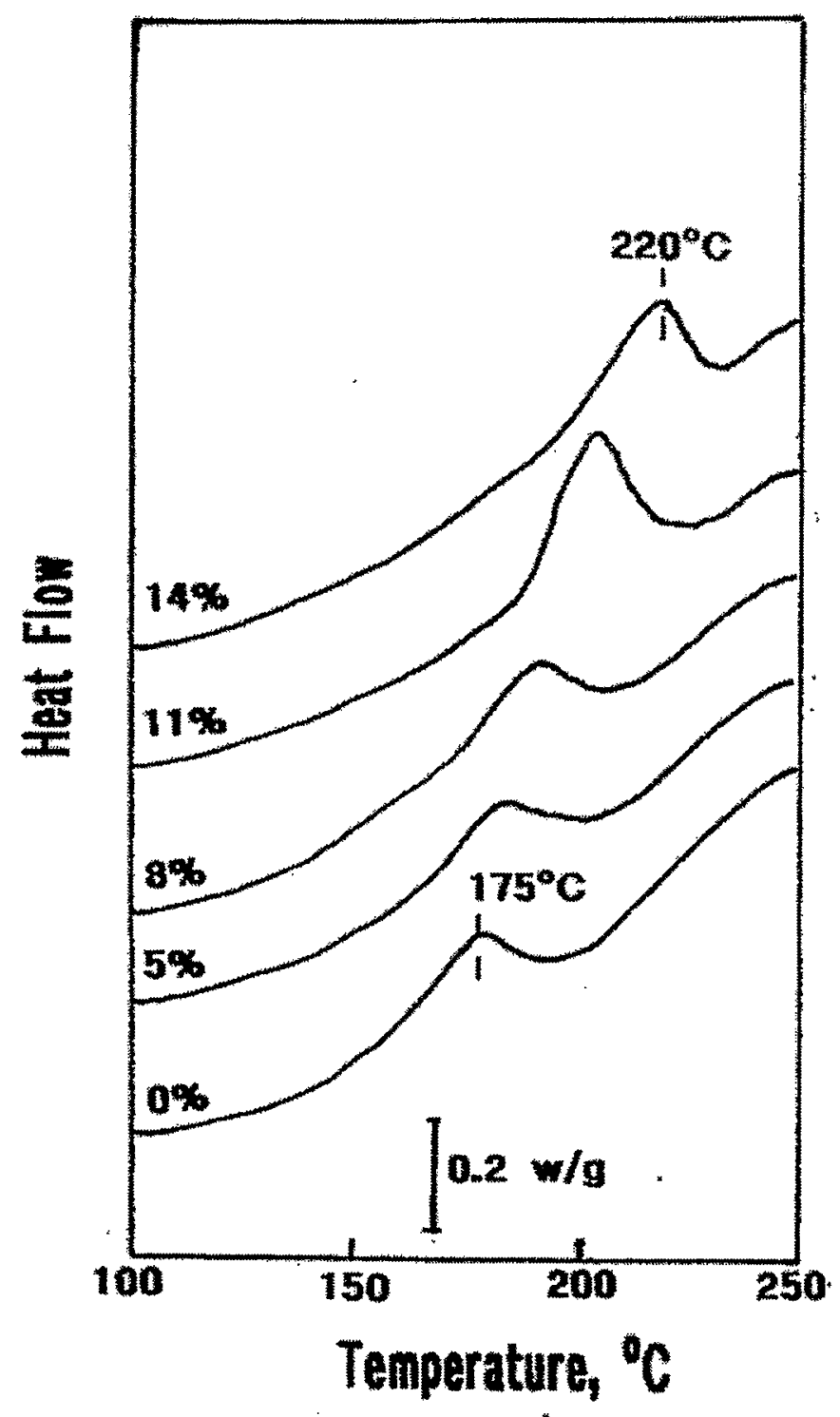

Figure 5. Changes in the exothermic crystallization temperature of PPS as a function of the content of treated MMT. 


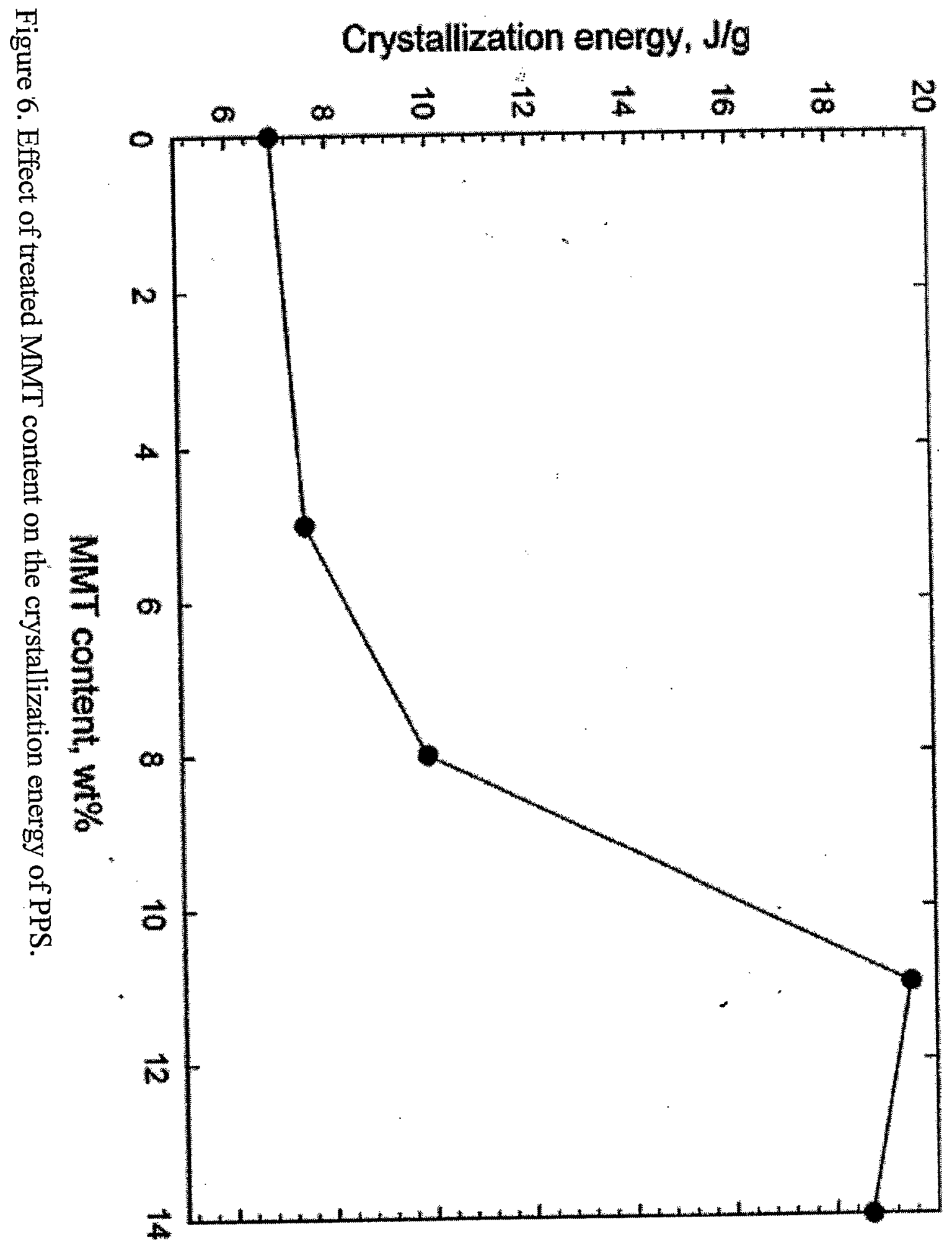




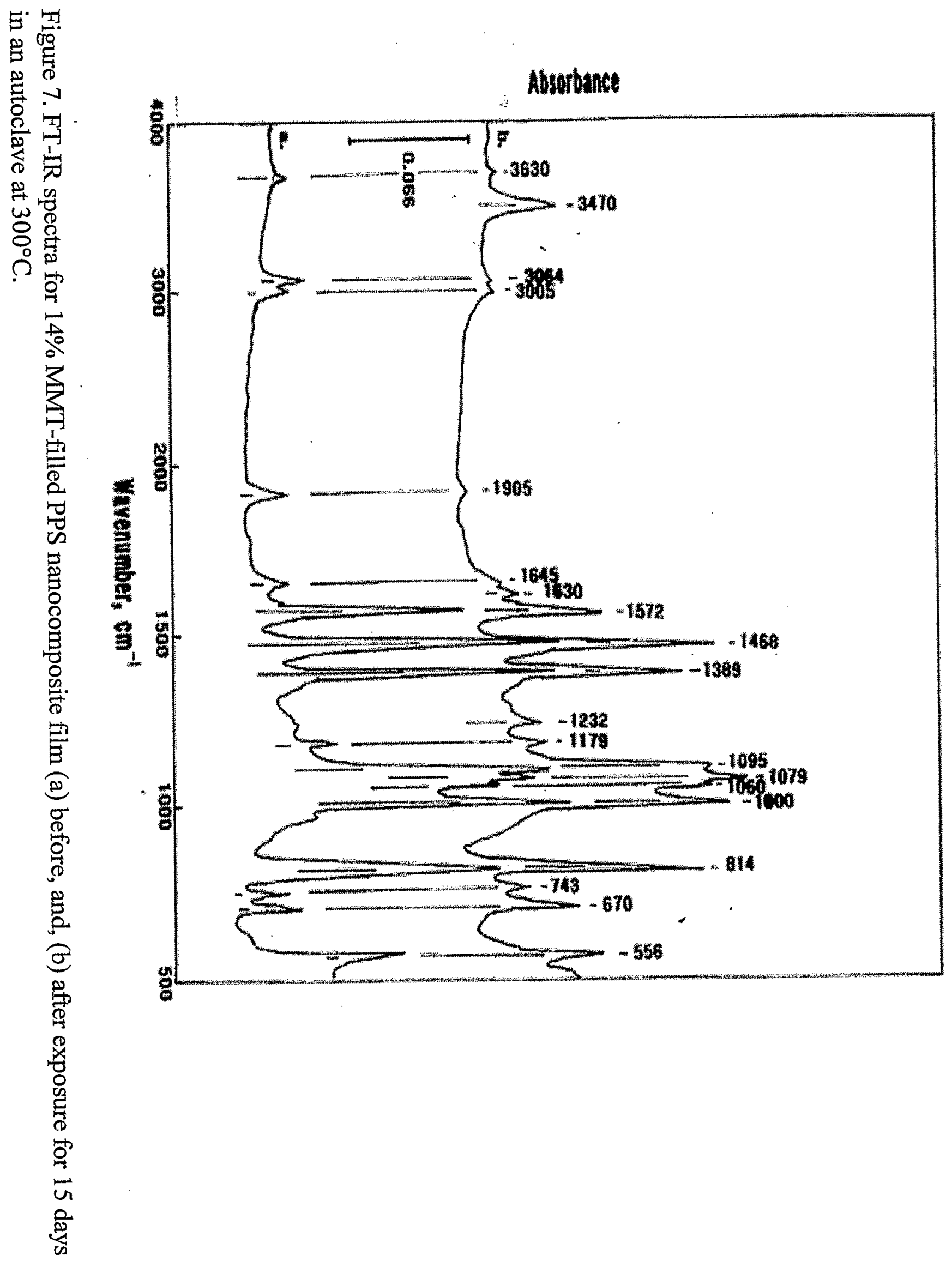




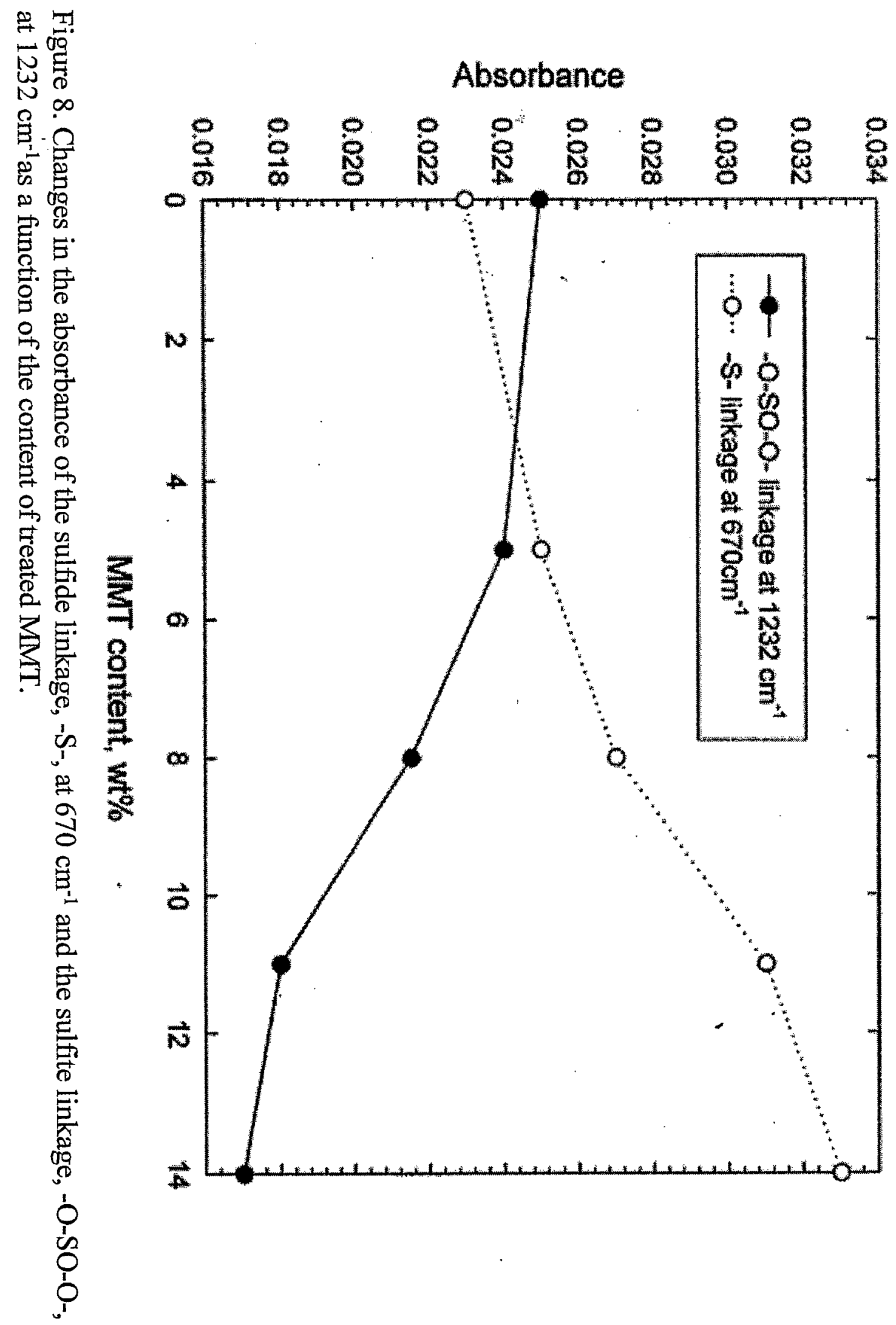




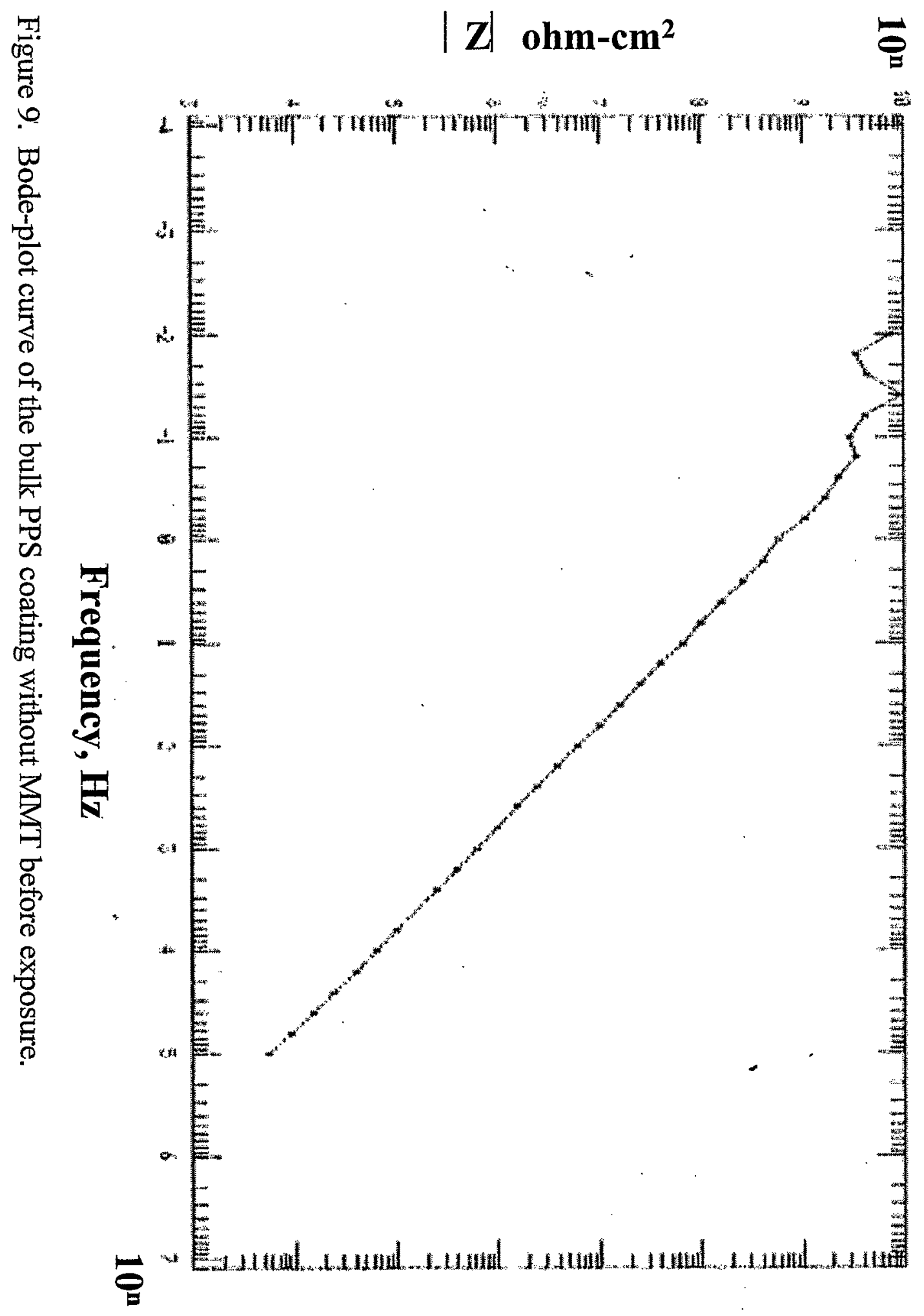




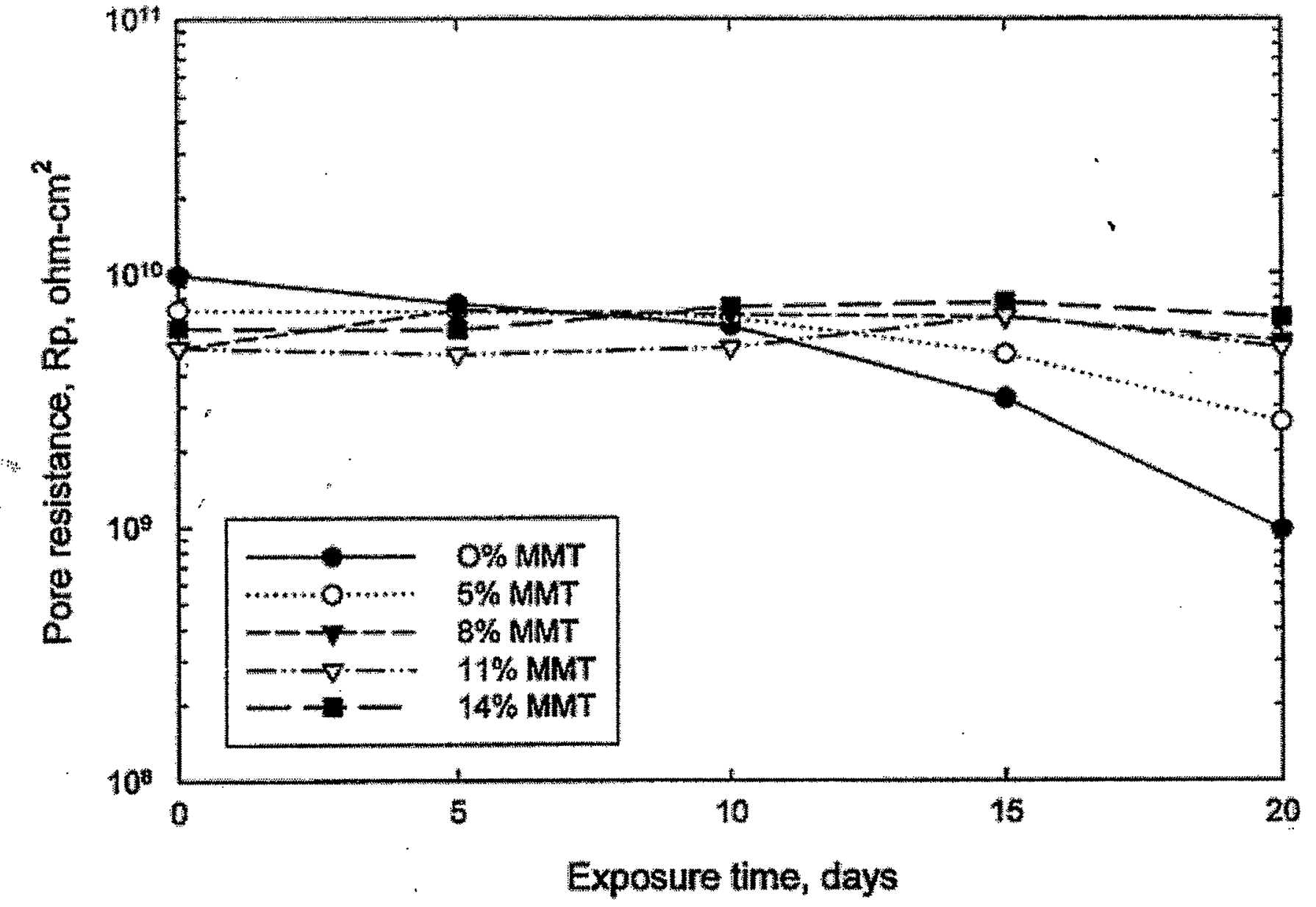

Figure 10. Pore resistance, Rp, of the PPS coatings containing $0,5,8,11$, and $14 \mathrm{wt} \%$ treated MMT after exposure for up to 20 days in a $300^{\circ} \mathrm{C}$ brine. 


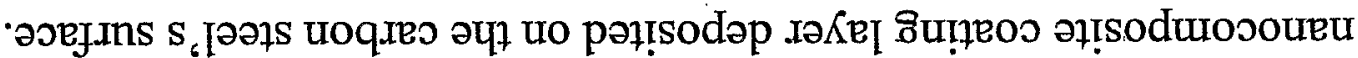

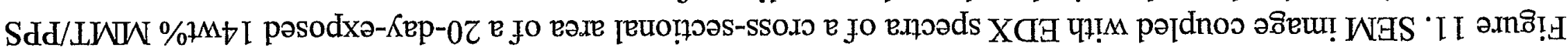

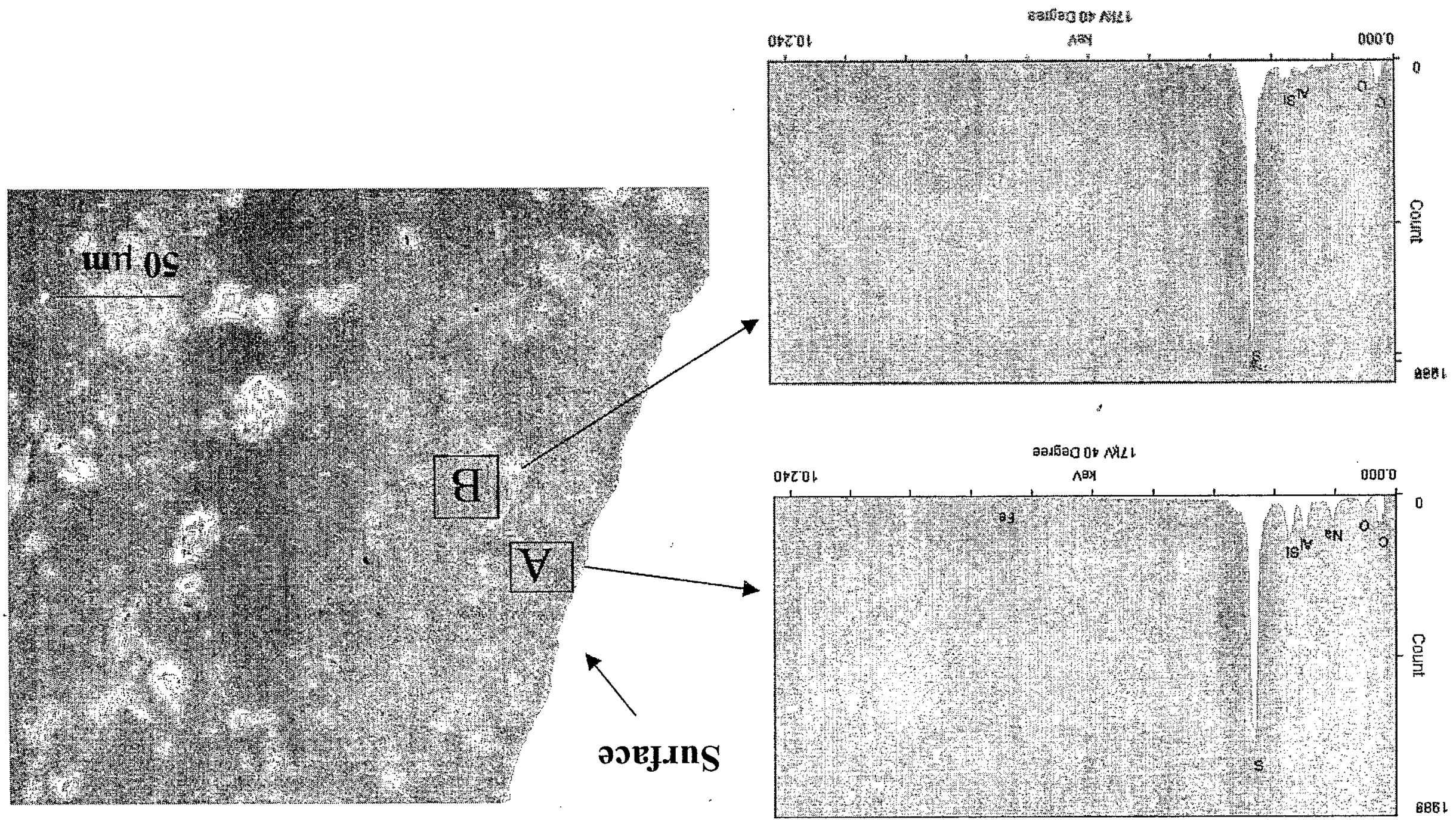




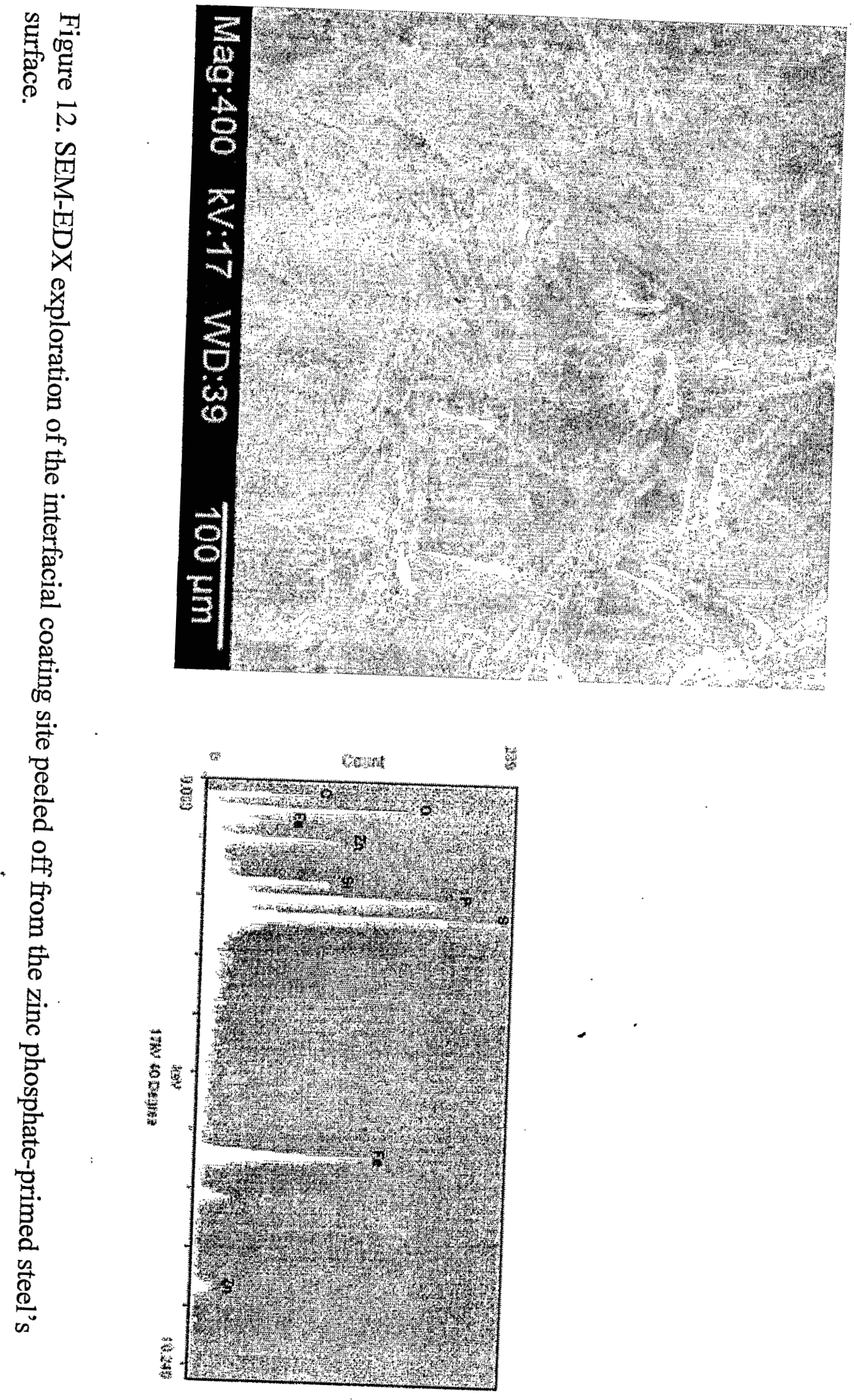

